\title{
DESHIDRATACIÓN Y ELABORACIÓN DE UN CONDIMENTO EN POLVO EN BASE A ORÉGANO (Origanum Vulgare L.)
}

\section{RESUMEN}

El presente trabajo es del tipo tecnológico; se determinó el mejor proceso de deshidratación y elaboración de condimentos a base de orégano, se hizo pruebas de aplicación práctica del condimento en pizzas: masa pizzera, extensión de masa, conformación, reposo, cocción, segmentación.

Para fijar el color se utilizó antioxidante ácido ascórbico, resultando la concentración óptima de $0,10 \%$. Es importante la temperatura de secado, siendo la más favorable $40^{\circ} \mathrm{C}$ y $50^{\circ} \mathrm{C}$. Las proporciones de orégano-aji fueron: $M_{1}=85 \%$ $15 \% ; M_{2}=80 \%-20 \% ; M_{3}=75 \%$ y $25 \%$; y $M_{4}=70 \%$ $30 \%$, resultando la más favorable la $\mathrm{M}_{2}$. Respecto a las mejores calificacionesan organolépticas de apariencia general en aroma, la mejor calificación la tiene la mezcla $\mathrm{M}_{4}$ y en consistencia no hay diferencias significativas; la calificación promedio es 4,75 .

\begin{abstract}
This work is sort of technology; was determined the best process of dehydration and development of condiments based oregano was evidence of practical application of condiment in pizza: mass pizza, extending mass, shape, resting, cooking, segmentation.
\end{abstract}

To set the color was used antioxidant ascorbic acid is the optimal concentration of $0.10 \%$. It is important temperature drying; being the most favourable $40 \mathrm{C}$ and $50^{\circ} \mathrm{C}$. The proportions were pepper-oregano: $M_{1}=85 \%-15 \% ; M_{2}=80 \%-20 \%$; $\mathrm{M}_{3}=75 \%$ and $25 \%$; and $\mathrm{M}_{4}=70 \%-30 \%$ resulting in the most favorable $\mathrm{M}_{2}$ regard to best organoleptic ratings of overall appearance in the aroma has the best rating the mixture $\mathrm{M}_{4}$ and consistency no significant differences, the average rating is 4,75 .

\section{INTRODUCCION}

El departamento de Tacna es el principal centro de producción de orégano, siendo las zonas productoras la sierra tacneña como Susapaya, Tarata, Candarave y Camilaca.

En nuestro medio el orégano es utilizado en forma directa en la preparación de platos alimenticios, y otra parte de la producción es destinada a la exportación pero en forma de materia prima.

Hasta la fecha este producto no tiene un valor agregado, no se conocen tecnologías para su aplicación industrial a pesar de que se sabe que el orégano tiene múltiples usos y aplicaciones, siendo uno de ellos el uso como condimento, más aún en estos tiempos en que se buscan productos de aplicación práctica, rápida y efectiva.

El presente trabajo de investigación busca obtener un condimento en polvo a base de orégano que tenga un uso rápido y práctico en la preparación de otros alimentos o productos. Para dicho fin se han trazado los siguientes objetivos:

1. Caracterización físico-química del orégano proveniente de los valles interandinos de Tacna.

2. Determinar el flujo más adecuado para el deshidratado del orégano y su posterior molienda.

3. Formular la mejor mezcla de insumos para obtener un condimento a base de orégano.

4. Estudio de las características organolépticas del uso práctico del condimento de orégano en la preparación de productos.

\section{MATERIALES Y MÉTODOS}

La investigación se realizó en los laboratorios de la Facultad de Ingeniería en Industrias Alimentarias, UNJBG, en 2 etapas.

- Caracterización de materia prima y deshidratación antes de su molienda.

- Formulación y procesos de aplicación del condimento seleccionado.

\section{Materias Primas}

La principal materia prima utilizada fue el orégano (Origanum vulgare), procedente de 
la provincia de Candarave, distrito de Talaca, sub. región Tacna. El otro insumo complementario es el aji amarillo seco procedente de los valles del distrito de Ite.

\section{Equipos,Materiales y Reactivos}

Equipos: Equipo deshidratador; laboratorio de operaciones unitarias e Ingenieria de Alimentos FAIA; horno de panificación, marca NOVA, 10 latas de capacidad; balanza analítica marca Sartorius, capacidad $160 \mathrm{~g}$. $0,0001 \mathrm{~g}$. de precisión; medidor de humedad; marca Memmert; equipo extractor Soxhlet; microdigestor, cabina quimica marca Corruma; reactivos: etanol $95 \%$ rojo fenol 0,4 $\mathrm{g} / \mathrm{l}$ en etanol al $20 \%$ hidróxido de potasio 0,5 $\mathrm{N}$; ácido sulfúrico, sulfato de cobre y potasio, hidróxido de sodio al $100 \%$, ácido bórico al $4 \%$, ácido clorhidrico al $0,1 \%$ silica gel.

\section{Metodología}

Diseño Experimental: El presente trabajo tuvo como diseño experimental el presentado en la Figura 3; para dicho fin se estudiaron las variables (), concentración de ácido ascórbico, la temperatura de deshidratación (T) y las mezclas de formulación (M).

\section{Procesamiento de Materia Prima:}

- Orégano : la materia prima principal una vez deshidratada, según el plazo de proceso seleccionado será sometido a una reducción de tamaño para tener lista para su aplicación y formulación de condimento.

- Aji Seco : se utilizará como complemento del orégano, para dicho fin de dispondrá de este insumo ya deshidratado; de igual modo sufrirá una reducción de tamaño antes de su incorporación a la formulación.

- Una vez definidas las posibles proporciones porcentuales de los insumos, estos serán mezclados y presentados bajo un solo contenido listo para su utilización práctica.

Fig. 03. Diseño Experimental Elaboración de un condimento a base de orégano

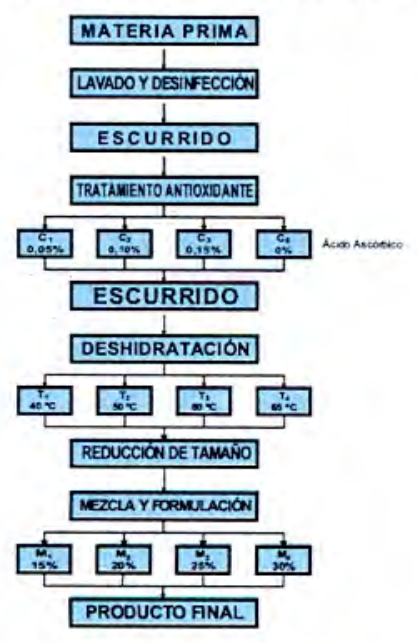

Metodos de Control. Los métodos de control seguidos son los normados según INDECOPI, siendo los principales los siguientes:

- Análisis químico proximal de la materia prima.

- Control y evaluación del proceso de deshidratación.

- Control y evaluación de molienda.

- Control de formulación y producto terminado.

\section{RESULTADOYDISCUSION}

1. Materia Prima: Para efectos de realización de pruebas de secado se dispuso de 5 kilos de orégano fresco procedente de la provincia de Candarave, distrito de Talaca.

Se hicieron cuatro pruebas de secado para determinar el mejor proceso de deshidratación.

Cuadro 01: Composición química proximal del orégano fresco.

\begin{tabular}{|c|c|}
\hline COMPONENTES & CONTENIDO \% \\
\hline 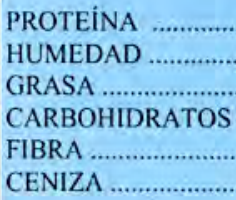 & 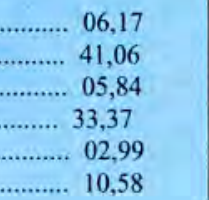 \\
\hline
\end{tabular}

Fuente : Elaboración propia

Del Cuadro $N^{\circ} 1$ se puede deducir que los contenidos de carbohidratos, humedad, proteina y ceniza son los mayoritarios, lo cual nos indica que aparte de sus aportes sensoriales como condimento pueden considerarse como fuente de fibra y carbohidratos para la nutrición.

Los datos obtenidos en el presente trabajo están dentro de los rangos reportados por la tabla de composición de alimentos del Ministerio de Salud (2002).

\section{Procesos de Deshidratación:}

La materia prima en proceso antes de ser sometida a deshidratación fue lavada y desinfectada en agua corriente y $1 \%$ de hipoclorito y, luego de un escurrido, sufrió un proceso de inmersión en solución de ácido ascórbico al $0,05 \%, 0.10 \%, 0,15 \%$ y $0 \%$, siendo la finalidad estabilizar el color y su rápida oxidación al ser expuesto al medio ambiente. Los resultados se reportan en el cuadro $\mathrm{N}^{\circ} 2$.

Cuadro 02: Tratamiento con solución antioxidante del orégano. 


\begin{tabular}{|c|c|c|}
\hline DENOMINACÓN & CONCENTRACION & OBSERVACIOS \\
\hline $\bar{c}_{t}$ & 0.05 & $\begin{array}{l}\text { Lipera variacion de } \\
\text { colonacion }\end{array}$ \\
\hline$\overline{\mathrm{C}_{2}}$ & 0,10 & $\begin{array}{l}\text { Color casis constante propio } \\
\text { del producto }\end{array}$ \\
\hline $\mathrm{C}_{3}$ & 0,15 & $\begin{array}{l}\text { Coloracióón ligeramente } \\
\text { intensa }\end{array}$ \\
\hline$\overline{\mathrm{C}_{\mathrm{a}}}$ & 0,00 & $\begin{array}{l}\text { Color muy intenso, verde } \\
\text { oscuro. }\end{array}$ \\
\hline
\end{tabular}

Fuente : Elaboración propia

Según los resultados, lo que interesa es que el producto mantenga su coloración verde, propio de la materia prima; esto se puede constatar después del escurrido y oreo. Pudiendo notarse que el antioxidante activa cualquier concentración aplicada, pero los mejores resultados se obtienen cuando se aplica $0,10 \%$, y cuando no se aplica antioxidante al orégano, pierde su color tendiendo a la oxidación, la fijación de color se mantiene hasta después del deshidratado.

Cuadro 03: Proceso de deshidratación del

\begin{tabular}{|c|c|c|c|c|c|c|c|c|}
\hline & & & & oc & & $s \sigma c$ & & \\
\hline & & 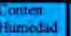 & 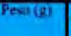 & $\begin{array}{l}\text { Conten } \\
\text { Humeas }\end{array}$ & & 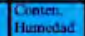 & & 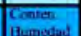 \\
\hline & 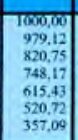 & $\begin{array}{l}37,42 \\
31,76 \\
252,27 \\
19.37 \\
15.46 \\
13,47\end{array}$ & 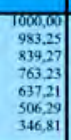 & $\begin{array}{l}36.24 \\
30.76 \\
23,727 \\
18.89 \\
14,43 \\
12,81\end{array}$ & 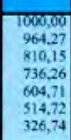 & 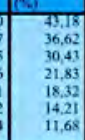 & 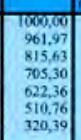 & 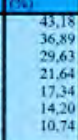 \\
\hline
\end{tabular}

\section{Fuente : Elaboración propia.}

Se puede indicar que se trata de una materia prima que no necesita del uso de temperaturas elevadas (convencionales 60$70^{\circ} \mathrm{C}$ ), sólo bastan temperaturas de entre 40 a $45^{\circ} \mathrm{C}$, lo cual nos permite un mayor manejo del secado y la obtención de adecuada humedad final $(10-12 \%)$. Temperaturas mayores permiten productos muy quebradizos y posiblemente cierta cocción que pueden atentar en contra de los componentes volátiles del orégano el cual es la razón de su uso como condimento.

3. Procesos de Molienda: Se hizo uso de un mortero y con la ayuda de un mazo se redujo de tamaño el orégano, y para uniformizar el tamaño se hizo pasar por un tamiz $\mathrm{N}^{\circ} 300$, siendo este tamaño el característico del orégano cuando es usado como condimento en la preparación de comidas, tales como guisos, estofados y pastas tipo pizza. De igual modo se procedió con el ají.

4. Mezclas y Formulaciones: Al final debe obtenerse un producto de aplicación práctica e inmediata, se tuvo que formular las mezclas de los insumos.

Cuadro 04 : Mezcla de insumos para la obtención de condimento a base de orégano.

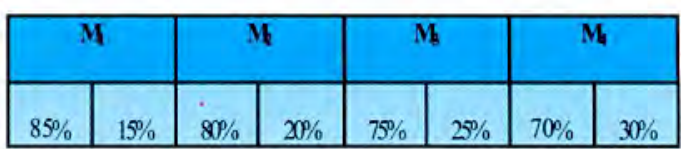

Fuente : Elaboración propia.

Luego de pasar una encuesta para evaluar las características organolépticas se encontró que la dispersión de observaciones se encuentra hacia los lados opuestos; quiere decir que algunos perciben el sabor picante y les agrada; otros no lo detectan y prefieren picante, aunque en esta oportunidad no se dispuso de otro que sea muy picante.

\section{Procesos de Elaboración del Producto:}

El presente trabajo tuvo su aplicación práctica en el uso del condimento en la preparación de PIZZA a la cual luego de agregar la guarnición se aplicó el condimento a base de orégano para luego someterlo a un panel para evaluar su aceptabilidd y juzgar las características organolépticas del producto.

\section{Fórmula 1:}

$300 \mathrm{~g}$. harina

$30 \mathrm{~g}$. de levadura

agua tibia (cantidad necesaria)

$15 \mathrm{~g}$. de sal

\section{Formula 2:}

$350 \mathrm{~g}$. harina

$25 \mathrm{~g}$. de levadura fresca o 15 de levadura prensada

1 huevo

una porción de leche tibia

\section{Producto Terminado y Evaluación} Organoléptica: El producto una vez obtenido fue sometido a un panel para su degustación y emita su opinión respecto a las caracteristicas organolépticas y se pueda juzgar las bondades del producto. Para dicho fin se utilizó el siguiente formato :

Ficha de Evaluación Organoléptica de un producto Pizza utilizando condimento a Base de Orégano.

Producto:

Fecha

Hora

Mediante la calificación indicada indique su puntuación a los atributos siguientes:

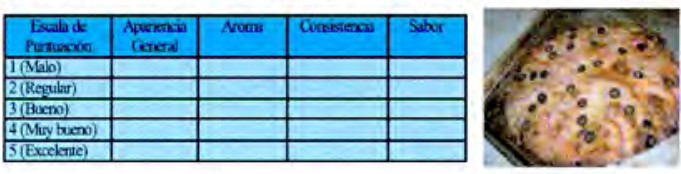

Evaluación de Apariencia General: Según los resultados, encontramos que aparentemente todas las muestras tienen 
buena calificación entre $4,4,5$ y 5 . La apariencia general involucra forma, tamaño, integridad, uniformidad. Este atributo depende de una buena conformación y distribución de componentes.

- Evaluación Aroma: Este atributo evalúa que resalte el olor a orégano, siendo éste un componente muy importante para este tipo de productos cuyas cantidades dependen del tamaño de la pizza, es decir, de la superficie circular a ser decorada. La muestra $\left(\mathrm{M}_{2}\right)$ es la que obtuvo las mejores calificaciones $(4,70)$.

Se puede indicar que la muestra cuyo componente mayoritario fue, orégano (30\%) resultó mejor el aroma y, por lo tanto, el gusto. Las calificaciones mejores están entre 4,6 y 4,5; salvo algunos casos entre 3,5 y 4,0.

- Evaluación de Consistencia: Percibe una mejor calificación teniendo en cuenta la suavidad al ser mordida, al ser cortada con un cuchillo y tenedor; debe ser fácil la operación y sobre todo que la guarnición no se destruya, asimismo que permita una buena masticación para apreciar su calidad gustativa. La calificación promedio alcanzada fue 4,75.

- Evaluación del Sabor: Segun los resultados obtenidos, de acuerdo con el cuadro $\mathrm{N}^{\circ} 8$, se encuentra que la mayoría de panelistas señalan que el producto muestra un excelente sabor y el $95 \%$ lo juzga con un calificativo de 5 , especialmente en la muestra $\left(M_{2}\right)$. Cabe indicar que para este tipo de producto el sabor debe ser agradable, muy exquisito al paladar, que permita saborear la combinación de ingredientes y a nuestro entender, el complemento utilizando ají mejora esta cualidad.

\section{CONCLUSIONES}

1.El tratamiento del orégano con sustancias antioxidantes como el ácido ascórbico previenen el deterioro de la materia prima vía oxidación, permitiendo a su vez fijar el color.

2.El secado artificial en cabina permita una deshidratación gradual y uniforme para el orégano; se logra buenos resultados con temperaturas de $40^{\circ}-45^{\circ} \mathrm{C}$ por 3 horas.

3.Los niveles de humedad para productos como el orégano son adecuados cuando éstos están entre $12-13 \%$, permitiendo su fácil y adecuado fraccionamiento y molienda.

4. La mejor mezcla para formular el condimento a base de orégano es de $75 \%$ de orégano y $25 \%$ de aji, el cual contribuye a mejorar las características organolépticas del producto pizza.

5.El panel de degustación conformado por 10 jueces dio el mejor puntaje a la muestra $2\left(\mathrm{M}_{2}\right)$ con calificativos de 4,74 respecto a la apariencia general, aroma, consistencia y sabor.

\section{RECOMENDACIONES}

Difundir y promover el uso del oregano como condimento, aconpañado de otras espeserias para mejorar las caracteristicas organolecticas de los productos.

Realizar otros estudios para la elaboracion de otros insumos a base de oregano y que tengan aplicación práctica y efectiva en la preparación de alimentos.

\section{BIBLIOGRAFIA}

Delgado de la Flor F. Voas (1996). Manual del Cultivo de hierbas de sabor. Instituto de Comercio Exterior, Lima.

Diagnostico de Áreas de Cultivo Tarata (1989), Programa de Desarrollo microregional Tacna.

SENATI, Perú (1998) Panadería Industrial zonal. Centro IDENPEI, Lima.

$\mathrm{h}$ ttp://www.agrogestión.el/imagen_agro/privado: /mercado 2005.pdj.

Http://www.mincetur.gob.pe/regiones/tacna/pdj $\mathrm{Http}: / / \mathrm{www}$.agritacna.gob.pe/documentos,apc/n oticias/items/rueda,negocios_oregano.pdf.

Http://www.inta.gov.or/altovalle/info/biblio/rompe Cabezas/pdfs/fyd 47 oreg. Pdf seard $=\% 22$ usoindustrial $\% 20$ de $\% 2$ orégano $\% 20$ ( condimento) $\% 22$.

\section{ANEXOS}

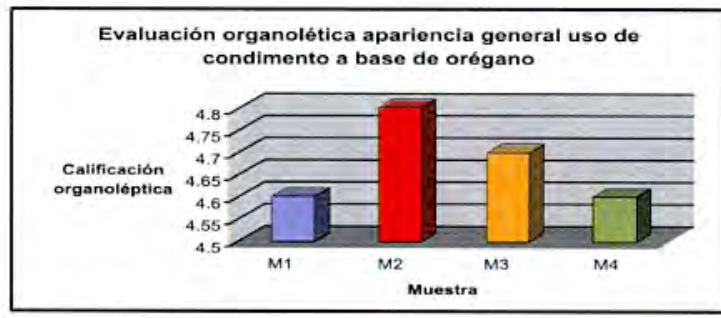

Evaluación organoléptica aroma uso de condimento a base de orégano

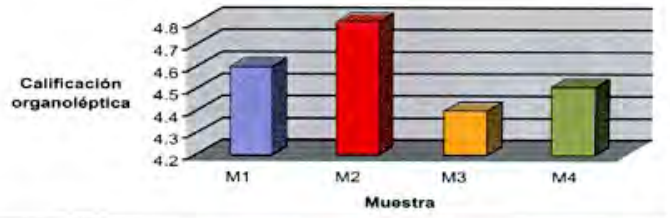

Evaluación organoléptica sabor uso de condimento a base de orégano

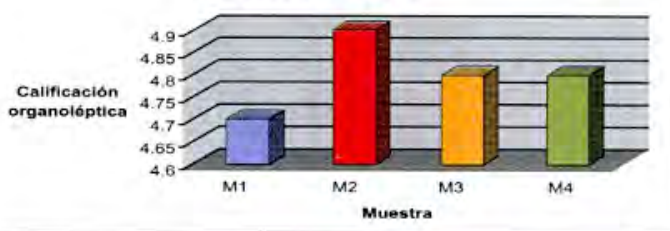

